\title{
The influence of
}

\section{phenotypic and genetic} effects on maternal

\section{provisioning and offspring weight gain in mice}

\author{
Reinmar Hager* and Rufus A. Johnstone \\ Department of Zoology, University of Cambridge, Cambridge \\ CB2 3EF, UK \\ *Author and address for correspondence: Faculty of Life Sciences, \\ University of Manchester, Manchester M13 9PT, UK. \\ (reinmar.hager@cantab.net).
}

Close interactions between mother and offspring are said to result in a coevolution of parental and offspring genotypes such that offspring are adapted in their solicitation behaviour to obtain maternal provisioning that maximizes their fitness. Few empirical studies have been conducted in this field and it remains unclear whether maternal provisioning and offspring weight gain are influenced by the same set of maternal and offspring phenotypic and genotypic factors. Using a cross-foster, splitlitter design in mice, we found that overall maternal provisioning and offspring weight gain are significantly correlated but are affected by a different set of parameters, except for the effect of maternal bodyweight. While the level of maternal provisioning was influenced by both offspring and foster mother genotype, offspring weight gain was only affected by the number of males in the mixed litter. We suggest that this disparity may hint at the inefficiency of offspring solicitation behaviour or effects of sibling competition.

Keywords: mice; maternal provisioning; offspring solicitation; coadaptation

\section{INTRODUCTION}

Offspring fitness is affected by genes expressed in both male and female parents as well as by maternally and paternally inherited genes expressed in offspring because parental traits influence the environment in which offspring grow up (Bateson 1994; Wolf \& Brodie 1998). This close interaction between young and parent is thought to lead to a coevolution of parental and offspring traits affecting growth and provisioning, consequently, any investigation of the genetic basis of parental investment must analyse the interactions between offspring and parental genotypes (Wolf et al. 1998). Moreover, studies of parent-of-origin-specific effects such as genomic imprinting have shown that maternally and paternally inherited genes may have different effects on an individual's phenotype, thus differentially affecting own fitness and that of related individuals uk.

(Haig 1997). Since these effects have been shown to play a crucial role in mammalian maternal resource transfer (Curley et al. 2004), it seems important to consider parent-of-origin specific effects when analysing the genetic basis of maternal provisioning and the interactions between offspring and parental traits. While several theoretical studies have modelled the coevolution of offspring and parental traits (e.g. Wolf 2000), most empirical studies in this field have focused on bird and insect systems (Kölliker et al. 2000; Agrawal et al. 2001; Lock et al. 2004).

In this study, we explore the genetic and phenotypic factors that influence both maternal provisioning and offspring fitness in a mammalian model system, the mouse (Mus musculus). Further, we advance on previous studies by including effects of sibling interactions and maternal genotype in our study. We investigate whether the presence of different-genotype litter mates affects overall maternal provisioning of the litter, thus influencing the effectiveness of offspring solicitation behaviour in mixed litters.

\section{MATERIAL AND METHODS}

Sexually mature mice of two strains (C57/B16 and $\mathrm{CBA} / \mathrm{Ca}$ ) were mated such that the timed birth of litters allowed cross-fostering pups of the required offspring genotypes (see Hager \& Johnstone 2003, 2005 for details). Building on this previous work we created mixed litters consisting of two half litters of the same paternal strain and either the same or different maternal strain. This allowed us to analyse whether offspring maternal genotype in mixed litters influences maternal provisioning and offspring solicitation behaviour. Upon birth, pups were marked and 82 mixed litters of a standard size of six or eight individuals created. Then, the resulting mixed litters were cross-fostered to females of either strain (foster mothers) that had given birth on the same day. All pups were cross-fostered on day 1 of their life. In total, the experiment yielded 12 different foster mother/offspring genotype combinations (see table 1).

Offspring weight gain and maternal provisioning were measured six days after delivery following our established procedure (Hager \& Johnstone 2003). We measured offspring and maternal weight change after a two hour period of nursing, which was preceded by a four hour simulated departure of the foster mothers. In our analysis we used General Linear Models (Minitab 13) with categorical and continuous variables as described in tables 3 and 4 (see electronic supplementary material). We checked the data for conformity with GLM requirements and error-structures for normality. The experiments described in this study comply with UK Home Office regulations, and did not require a license.

\section{RESULTS}

Confirming results of previous work (Hager \& Johnstone 2003), we found a significant effect of father's strain on litter size with CBA males siring larger litters than B6 males $\left(F_{1,81}=4.15, p=0.045\right)$, and a significant effect of female bodyweight on litter size $\left(F_{1,81}=5.78, p=0.019\right)$. Females of the two strains did not significantly differ in their bodyweights at the beginning of the experiment. However, 6 days after delivery, females that were mated to CBA males were significantly heavier ( $t$-test, $t=-2.62, p=0.011$, d.f. $=76)$ and also produced significantly larger litters ( $t$-test, $t=-2.39 ; p=0.019 ;$ d.f. $=76$ ). A female's bodyweight on day 6 was positively affected by the size of the litter she produced and that of her adoptive litter as well as by her strain (with B6 females being heavier; $F_{1,76}=21.43, p>0.001$, 
Table 1. Experimental design. (A mixed litter consisted of two half litters, each containing three or four pups. All pups in a mixed litter were of the same paternal strain. The first strain in a half litter refers to their biological mother's strain while the second denotes the father's strain.)

\begin{tabular}{llll}
\hline \multicolumn{4}{l}{ foster mother strain } \\
\cline { 3 - 3 } & & B6 & CBA \\
\hline father & CBA & B6CBA/B6CBA & B6CBA/B6CBA \\
strain & & B6CBA/CBACBA & CBACBA/B6CBA \\
& & CBACBA/CBACBA & CBACBA/CBACBA \\
& B6 & B6B6/B6B6 & B6B6/B6B6 \\
& & B6B6/CBAB6 & CBAB6/B6B6 \\
& CBAB6/CBAB6 & CBAB6/CBAB6 \\
\hline
\end{tabular}

natural litter size: $F_{1,76}=5.53 ; p=0.021$ and size of adoptive litter $F_{1,76}=4.81, p=0.031$ ).

\section{(a) Maternal provisioning}

First, we analysed the factors influencing female provisioning effort by focusing on the factors that influence foster mother's weight change over a two hour period nursing her adoptive mixed litter. GLM analysis incorporating offspring and parental genotypic and phenotypic traits as factors revealed that both foster mother and offspring genotype significantly affected the level of maternal provisioning (table 3, electronic supplementary material). Offspring genotype within a mixed litter was divided into the following three categories: (i) all pups were of B6 maternal genotype, (ii) one half litter was of B6 and the other of CBA maternal genotype and (iii) all pups were of CBA maternal genotype. This allowed us to analyse whether offspring of a specific genotype have a large influence on maternal provisioning and whether, for instance, pups in a mixed litter benefit from the presence of littermates that are better at soliciting maternal resources. Controlling for all other factors in the model, we found that mothers' bodyweight change was most negatively affected when she nursed a litter which consisted exclusively of pups whose mother was of the B6 strain (as indicated by the most negative of the three coefficients in table 3, electronic supplementary material). The level of bodyweight change was of similar magnitude when the mixed litter was composed of two half litters of dissimilar maternal genotype. Foster mothers showed the smallest change in bodyweight when all pups in the litter had a CBA mother. Over and above the offspring genotype effect, foster mother's bodyweight significantly influenced her weight loss with heavier females losing more weight than lighter females.

\section{(b) Offspring weight gain}

Providing maternal resources to offspring should be reflected in equivalent bodyweight gain of the young, and overall maternal provisioning and offspring weight gain were highly significantly correlated $\left(F_{1,75}=35.32 ; p<0.001, r^{2}=32.3\right)$.

However, the analysis of offspring weight gain shows that offspring maternal genotype and foster mother strain have no significant effect on this response variable (table 4, electronic supplementary material). While the strongest predictor was still foster mother bodyweight (such that litters that were fostered by heavier females gained more weight overall), the only other significant predictor was the sex ratio of the mixed litter. Litters that contained more males gained less weight. Over and above these main effects we found a significant interaction between foster mother strain and her mate's strain. If both of these were the same (i.e. either both CBA or both B6) the litter gained less weight. On the other hand, if foster mother genotype and that of her mate were different to each other, then the pups in the focal litter gained more weight.

To investigate potential effects of sibling competition, we compared the weight gain of pups in the three conditions as described in the previous section. From the above results we would expect that CBA maternal pups gained from the presence of B6 maternal pups in a mixed litter because maternal provisioning to such litters was almost the same as to litters consisting of B6 maternal pups only (which was much greater than to CBA only litters). However, we found only a non-significant trend when performing a GLM that compares the growth of CBA maternal pups in mixed litters with B6 maternal pups to that of CBA maternal pups in pure CBA maternal litters. A comparison of the effects of the predictor variables on maternal provisioning and offspring weight gain is given in table 2 .

\section{DISCUSSION}

Our analysis of maternal provisioning and offspring weight gain clearly shows that female bodyweight is the most important predictor of the two parameters. Everything else being equal, pups gained most weight when fostered by heavier females. While females of the two strains were mated at random with respect to their bodyweights and there was no significant difference in their bodyweight at the beginning of the experiment, six days after delivery females that were mated to CBA males were significantly heavier. An explanation for this finding may be that CBA males sired significantly larger litters and, as a result, the females that gave birth to a larger litter became heavier. Indeed, both the size of the natural litter as well as the size of the adopted litter positively affected female bodyweight on day 6 . This suggests that females may be primed to provision for a larger litter but that in addition they can put on extra weight when given a larger litter postnatally.

\section{(a) Factors infuencing offspring weight gain in mixed litters}

Surprisingly, litters that contained a higher proportion of males gained less weight. In mice males are larger than females and thought to be energetically more costly to produce due to their higher energy demands, however, sex ratio effects on bodyweight increase in mice do not show a clear pattern (Drickamer 1976). It seems plausible that our results may be due to increased levels of within-litter competition among males, suggesting that competition for access to teats 
Table 2. Summary of predictor variable effects on maternal provisioning and offspring weight gain. (For details of the statistical parameters please refer to tables 3 and 4 (electronic supplementary material). ' + ' indicates a positive effect while '-' refers to a negative effect. The sex ratio is calculated as the number of males over total litter size.)

\begin{tabular}{|c|c|c|}
\hline & $\begin{array}{l}\text { effects on } \\
\text { maternal } \\
\text { provisioning }\end{array}$ & $\begin{array}{l}\text { effects on } \\
\text { offspring } \\
\text { weight gain }\end{array}$ \\
\hline $\begin{array}{l}\text { foster mother bodyweight } \\
\text { foster mother strain }\end{array}$ & - & + \\
\hline B6 & - & ns \\
\hline $\mathrm{CBA}$ & + & ns \\
\hline \multicolumn{3}{|l|}{ offspring maternal genotype } \\
\hline B6X/B6X & - & ns \\
\hline B6X/CBAX & - & ns \\
\hline CBAX/B6X & - & ns \\
\hline CBAX/CBAX & + & ns \\
\hline litter size & ns & ns \\
\hline offspring bodyweight & ns & ns \\
\hline sex ratio & ns & - \\
\hline foster mother's litter size & ns & \\
\hline $\begin{array}{l}\text { foster mother strain } \times \text { mate } \\
\text { strain }\end{array}$ & ns & unclear \\
\hline
\end{tabular}

may be more costly and time consuming, thus leaving less time to suck.

In addition to the sex ratio effect, we found a negative effect on offspring weight gain if their foster mother was mated to a B6 male given she herself was of that same strain. The interaction hints at the possibility that female resource allocation may depend on the perceived quality of her mate as proposed by theories of differential allocation (Gowaty et al. 2003) or, alternatively, at effects of inbreeding depression (Falconer \& Mackay 1996). At present, however, it remains difficult to interpret the full implications of this interaction and requires further specifically designed experiments to elucidate.

\section{(b) Factors influencing maternal provisioning to mixed litters}

The levels of maternal provisioning were influenced by the mixed litters' maternal genotypes and were nonlinear for the three conditions. When females nursed a mixed litter that consisted exclusively of B6 maternal pups, maternal bodyweight was most negatively affected, which was only slightly different from when females nursed two half litters with dissimilar maternal genotype. Litters with only CBA maternal pups received the smallest amount of maternal provisioning.

This result can be interpreted in the light of potential differences between the strains either in their ability to elicit provisioning or in their competitiveness (e.g. in nipple shifting; Drewett 1983). It may be that pups whose mother was of a B6 strain are on average better at eliciting resources from foster mothers than CBA maternal pups, e.g. through teat stimulation or vocalizations. Even the presence of only a few B6 maternal pups in litters, in which the other half litter was CBA maternal, had virtually the same impact on maternal provisioning compared to litters of B6 only pups. This suggests that CBA maternal pups in mixed litters may benefit from the presence of a few 'greedy' pups although there was only a non-significant tendency for a higher weight gain of CBA maternal pups raised in litters with B6 maternal pups compared to those in CBA maternal only litters. Further behavioural studies are needed to assess whether B6 pups are more competitive or if they are better at occupying the more productive anterior teats (e.g. Barnard et al. 1998) yielding further insights into mother-offspring coadaptation.

Overall, maternal provisioning and offspring weight gain are significantly correlated. While both measures are chiefly explained by maternal bodyweight, we found that genetic factors such as genotype of a mixed litter have more subtle effects and are only detected when focusing on one of the parameters. This raises the question of whether the disparity observed in our study is due to the overall weaker effects of these parameters or whether short-term offspring weight gain and maternal provisioning are indeed governed by a different set of parameters. Sibling competition, the efficiency of growth or hybrid vigour are potential factors that could account for such a discrepancy.

We would like to thank Per Smiseth and two anonymous referees for helpful comments on an earlier version of this manuscript. This research was supported by the Royal Society and the Newton Trust.

Agrawal, A. F., Brodie, E. D. \& Brown, J. 2001 Parentoffspring coadaptation and the dual genetic control of maternal care. Science 292, 1710-1712. (doi:10.1126/ science.1059910)

Barnard, C. J., Behnke, J. M., Gage, A. R., Brown, H. \& Smithurst, P. E. 1998 Maternal effects on the development of social rank and immunity trade-offs in male laboratory mice (Mus musculus). Proc. R. Soc. B 265, 2087-2093. (doi:10.1098/rspb.1998.0544)

Bateson, P. 1994 The dynamics of parent-offspring relationships in mammals. TREE 10, 399-403.

Curley, J. P., Barton, S. C., Surani, M. A. \& Keverne, E. B. 2004 Co-adaptation in mother and infant regulated by a paternally expressed imprinted gene. Proc. R. Soc. B 271, 1303-1309. (doi:10.1098/rspb.2004.2725)

Drewett, R. F. 1983 Sucking, milk synthesis, and milk ejection in the Norway rat. In Parental behaviour of rodents (ed. R. W. Elwood), pp. 181-294. New York: Wiley.

Drickamer, L. C. 1976 Effect of size and sex ratio of litter on the sexual maturation of female mice. F. Reprod. Fertil. 46, 369-374.

Falconer, D. S. \& Mackay, T. F. C. 1996 Introdcution to quantitative genetics, 4 th edn. Harlow: Longman.

Gowaty, P. A., Drickamer, L. C. \& Schmid-Holmes, S. 2003 Male house mice produce fewer offspring with lower viability and poorer performance when mated with females they do not prefer. Anim. Behav. 65, 95-103. (doi:10.1006/anbe.2002.2026)

Hager, R. \& Johnstone, R. A. 2003 The genetic basis of family conflict resolution in mice. Nature 421, 533-535. (doi:10.1038/nature01239)

Hager, R. \& Johnstone, R. A. 2005 Differential growth of own and alien young in mixed litters of mice: a role for 
genomic imprinting? Ethology 111, 705-711. (doi:10. 1111/j.1439-0310.2005.01097.x)

Haig, D. 1997 Parental antagonism, relatedness asymmetries, and genomic imprinting. Proc. R. Soc. B 264, 1657-1661. (doi:10.1098/rspb.1997.0230)

Kölliker, M., Brinkhof, M. W. G., Heeb, P., Fitze, P. S. \& Richner, H. 2000 The quantitative genetic basis of offspring solicitation and parental response in a passerine bird with biparental care. Proc. R. Soc. B 267, 2127-2132. (doi:10.1098/rspb.2000.1259)
Lock, J. E., Smiseth, P. R. \& Moore, A. J. 2004 Selection, inheritance, and the evolution of parentoffspring interactions. Am. Nat. 164, 13-24. (doi:10. $1086 / 421444)$

Wolf, J. B. 2000 Gene interactions from maternal effects. Evolution 54, 1882-1898.

Wolf, J. B. \& Brodie, E. D. 1998 Coadaptation of parental and offspring characters. Evolution 52, 535-544.

Wolf, J. B., Brodie, E. D., Cheverud, J. M., Moore, A. J. \& Wade, M. J. 1998 TREE 13, 64-69. 